\title{
VARIATIONS DES CATECHOLAMINES \\ AU COURS DU CHOC DE L'HOMME
}

\author{
M. Hanquet, M.D., A. Cession-Fossion, M.D., et J. Lecomte, M.D. ${ }^{\circ}$
}

LES DERNIÈRES ÉTUDES EXPÉRIMENTALES consacrées au choc ont fortement modifié les conceptions jadis classiques de ce syndrome: à la lumière de leurs résultats, le choc n'est plus assimilable à un simple collapsus. Son diagnostic n'est plus tributaire de la seule chute de la pression artérielle générale, bien que nombreux sont encore ceux pour qui l'hypotension sévère en est un signe essentiel, et qui vont jusqu'à faire de celle-ci le facteur causal du choc, suivant l'exemple d'Archibald et MacLean. ${ }^{1}$

Qu’il soit de nature cardiogénique, neurogénique, hémorragique ou septique, le choc apparait aujourd'hui comme "une voie terminale commune"; c'est une réaction de défense, destinée à protéger par tous les moyens, fussent-ils préjudiciables à certaines fonctions, lirrigation du cœur et surtout celle du cerveau. ${ }^{2}$ Le facteur causal est un état de détresse circulatoire qui entraîne l'adaptation du contenant vasculaire à son contenu. La surface de laire vasculaire normale diminue selon les nécessités du moment et le débit sanguin, réduit, est alors réservé à la circulation cérébrale et à la circulation coronarienne. ${ }^{3}$ De cet état découle une symptomatologie connue de longue date, qui traduit un ralentissement circulatoire périphérique intense se manifestant par une pâleur de la peau, froide et cyanosée, par une réduction de la diurèse, par des perturbations respiratoires et, enfin, par diverses modifications de la pression artérielle dont le pincement de la différentielle est le signe le plus habituel.

Différents phénomènes peuvent avoir sur l'évolution du choc les plus graves effets: (a) Au niveau de la peau et des muscles, le ralentissement circulatoire provoque un état d'acidose métabolique, conséquence de l'insuffisance d'oxygénation tissulaire. (b) Au niveau du tube digestif, l'ischémie provoque la résorption de toxines microbiennes qui passent dans le système veineux portal. $(c)$ Le foie est l'objet de perturbations circulatoires qui, tout en provoquant des modifications métaboliques, l'empêchent de jouer un rôle normal de barrière aux toxines intestinales. $(d)$ La fonction rénale est réduite et, parfois, l'anurie s'installe.

Le mécanisme essentiel responsable de cette situation procède d'une vasoconstriction qui intéresse tous ces territoires et plus généralement les différents vaisseaux porteurs de récepteurs $\alpha$ adrénergiques. Ces derniers sont stimulés par les catécholamines que secrètement en abondance la médullo-surrénale et les terminaisons sympathiques, en réponse à l'état de détresse circulatoire aigüe initial."

Les phénomènes se déroulent en deux étapes successives appelées, par certains, shock réversible et choc irréversible. ${ }^{5,6}$ Au cours de la première étape, la vasoconstriction intéresse les sphincters artériels précapillaires et les sphincters veineux

\footnotetext{
*Avec la collaboration technique de R. Vandermeulen. Les autors sont du Service d'anesthésiologie et l'Institut Léon Frédéricq, Physiologie, Université de Liège.
} 
postcapillaires d'un vaste territoire vasculaire qui est ainsi isolé de la circulation centrale. Celle-ci, amputée d'une large partie des circuits qu'elle doit normalement alimenter, conserve un débit suffisant à l'irrigation myocardique et cérébrale. La pression systolique qui règne dans les vaisseaux centraux est maintenue, la pression diastolique est augmentée, la pression différentielle est pincée, mais l'irrigation des tissus périphériques devient insuffisante. C'est dans ces conditions que survient l'acidose métabolique ainsi que les perturbations fonctionnelles de l'intestin, du foie et surtout des reins, signalées plus haut. Progressivement apparaît la deuxième étape qui est caractérisée par un relâchement des sphincters artériels précapillaires alors que les sphincters veineux postcapillaires restent fermés. De ce fait, une quantité importante de sang s'engouffre dans le système capillaire primitivement isolé. La pression hydrostatique qui règne dans les capillaires s'élève jusqu'à atteindre des valeurs qui dépassent celles de la pression collö̈dosmotique du sang; du plasma passe alors en abondance dans les tissus interstitiels. La masse sanguine précaire qui maintenait artificiellement jusque là une pression systolique suffisante dans les vaisseaux centraux, diminue brusquement par perte de sang dans les capillaires dilatés, puis par fuite de plasma dans les tissus interstitiels: c'est l'effondrement circulatoire généralisé qui aboutit à l'état de choc irréversible et à la mort.

Plusieurs interprétations ont été données à cette différence de comportement des sphincters précapillaires et postcapillaires; parmi celle-ci, on évoque une sensibilité plus grande des sphincters artériels précapillaires à l'acidose, sur lesquels les catécholamines n'auraient plus une action suffisante.

A la lumière de ces différentes considérations sur le rôle des catécholamines, il nous a paru intéressant de doser l'adrénaline (A) et la noradrénaline (NA) au cours de chocs observés chez l'homme. Ainsi, nous avons dosé les catécholamines circulantes chez des blessés atteints de choc clinique grave et nous avons comparé les résultats obtenus à ceux que donnent les individus normaux. De même, nous avons mesuré les catécholamines des surrénales de blessés morts en état de choc en comparant les taux observés à ceux obtenus à partir des surrénales d'individus non choqués.

\section{Techniques}

\section{Dosage des catécholamines sanguines}

Sujets normaux. Vingt millilitres de sang sont prélevés chez 21 adultes normaux soumis à un régime hospitalier ambulatoire. Le sang est immédiatement additionné d'acide ascorbique $(0.5 \mathrm{ml}$ d'une solution à 002 pour cent $)$ dans le but d'éviter l'auto-oxydation des catécholamines. Il est ensuite centrifugé, l'hématocrite est mesuré et le plasma est récolté. Les amines qu'il contient sont dosées par la méthode fluorimétrique de von Euler et Lishajko; ${ }^{7}$ le plasma est passé sur colonne d'alumine à $\mathrm{pH} \mathrm{8.4,} \mathrm{en} \mathrm{présence} \mathrm{d'EDTA,} \mathrm{et} \mathrm{les} \mathrm{catécholamines} \mathrm{sont} \mathrm{éluées} \mathrm{par}$ l'acide acétique $0.25 \mathrm{~N}$. Elles sont oxydées par du $\mathrm{K}_{3} \mathrm{Fe}(\mathrm{CN})_{6}$ et transformées en lutines fluorescentes par un mélange de $\mathrm{NaOH}$ et d'acide ascorbique. Les mesures s'effectuent au spectrofluorimètre Zeiss.

Sujets en état de choc. La méthode de dosage est la même que chez les individus normaux. Les prélèvements sont effectués chez 14 patients hospitalisés au 
TABLEAU I

Concentration DEs catécholamines PLASMATIQUES EXPRIMEES EN $\mu \mathrm{g} / \mathrm{L}$

Plasma Catecholamine Concentrations, EXPRESSED IN $\mu \mathrm{g} / \mathrm{L}$ OF BLOOD

\begin{tabular}{cccc}
\hline No & A & NA & A + NA \\
\hline 1 & 1.24 & 2.55 & 3.79 \\
2 & 1.22 & 2.29 & 3.51 \\
3 & 2.32 & 2.74 & 5.06 \\
4 & 3.97 & 4.80 & 8.77 \\
5 & 1.83 & 2.95 & 4.78 \\
6 & 1.75 & 2.09 & 3.84 \\
7 & 0.52 & 1.82 & 2.34 \\
8 & 1.34 & 1.85 & 3.19 \\
9 & 2.31 & 3.07 & 5.38 \\
10 & 1.43 & 1.90 & 3.33 \\
11 & 2.62 & 3.58 & 6.19 \\
12 & 1.24 & 2.59 & 3.83 \\
13 & 1.33 & 1.64 & 2.97 \\
14 & 1.11 & 2.15 & 3.26 \\
15 & 1.26 & 1.51 & 2.77 \\
16 & 1.25 & 1.63 & 2.88 \\
17 & 1.31 & 1.57 & 2.88 \\
18 & 1.30 & 2.42 & 3.72 \\
19 & 1.25 & 2.46 & 3.71 \\
20 & 1.71 & 2.23 & 3.94 \\
21 & 0.89 & 1.44 & 2.33 \\
Moyenne & 1.58 & 2.35 & 3.93 \\
\hline
\end{tabular}

Centre de réanimation du Service de chirurgie de l'Hôpital de Bavière pour des affections diverses, le plus souvent traumatiques, ayant entraîné un état de choc cliniquement indiscutable. Plusieurs prélèvements sont effectués chez le même patient, si possible jusqu'au moment de la dissipation clinique du choc.

\section{Dosage des catécholamines surrénaliennes}

Surrénales normales. Elles sont prélevées chez des individus atteints d'artérite oblitérante dont le fonctionnement cardiovasculaire était par ailleurs normal et pour lesquels une surrénalectomie était pratiquée à titre thérapeutique sous anesthésie générale. Dès leur prélèvement, les surrénales ont été soigneusement nettoyées, pesées et broyées dans l'acide trichloracétique à 5 pour cent. Leur teneur en catécholamines est déterminée par spectofluorimétrie selon la technique de von Euler et Lishajko. ${ }^{7}$

Surrénales prélevées en cours de choc. Elles ont été prélevées chez des individus morts en état de choc, immédiatement après le décès. Elles ont été traitées comme ci-dessus.

\section{Résultats et Discussion}

\section{Catécholamines sanguines}

Le Tableau I reprend les valeurs des catécholamines sanguines chez les individus normaux, exprimées en $\mu \mathrm{g} / \mathrm{L}$ de sang. La teneur en noradrénaline est toujours supérieure à la teneur en adrénaline et, en général, la somme des deux catécholamines reste inférieure à $4 \mu \mathrm{g} / \mathrm{L}$ (dans 16 cas sur 21). 
Le Tableau II reprend outre les valeurs des catécholamines observées chez les patients en état de choc, les causes de choc, l'âge des patients, leur état circulatoire et leur diurèse horaire. Chez 14 patients, on relève: cinq hémorragies intrapéritonéales d'origine traumatique (rupture du foie, de la rate ou d'un viscère creux), cinq hémorragies rétropéritonéales par fracture du bassin, une hémorragie externe importante, une hémorragie gastrique, une fracture du crâne avec réaction neurovégétative sévère, un choc septique.

\section{TABLEAU II}

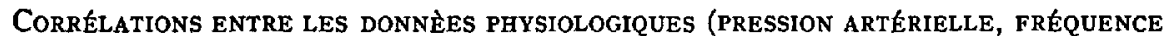
CARDIAQUE ET DIURÈSE) ET LA TENEUR EN CATÉCHOLAMINES

Correlations between Physiological Parameters (Arterial Bloon Pressure, Cardiac Rate, and Hourly Urine Output) and Plasma Catecholamines

\begin{tabular}{|c|c|c|c|c|c|c|c|}
\hline \multirow{2}{*}{$\begin{array}{l}\text { Patien } \\
N^{\circ}\end{array}$} & \multirow[b]{2}{*}{ Diagnostic } & \multirow[b]{2}{*}{ Age } & & \multicolumn{3}{|c|}{ Etat de choc } & \multirow{2}{*}{$\begin{array}{l}\text { Choc } \\
\text { terminé }\end{array}$} \\
\hline & & & & $1^{\text {re }} \mathrm{h}$. & $2^{\mathrm{me}} \mathrm{h}$. & $3^{\text {mo }} h$. & \\
\hline 1 & $\begin{array}{l}\text { Fractures multiples } \\
\quad \text { (bassin) }\end{array}$ & 20 & $\begin{array}{r}\text { a } \\
\mathbf{b} \\
\mathbf{c} \\
\text { ad. } \\
\text { nad. } \\
\text { nad. + ad. }\end{array}$ & $\begin{array}{l}12 / 7 \\
90 \\
100 \\
1.63 \\
2.55 \\
4.18\end{array}$ & $\begin{array}{l}14 / 7 \\
100 \\
30 \\
1.21 \\
2.51 \\
4.72\end{array}$ & $\begin{array}{c}14 / 7 \\
120 \\
30 \\
- \\
-\end{array}$ & $\begin{array}{l}15 / 8 \\
100 \\
50 \\
0.94 \\
2.01 \\
2.95\end{array}$ \\
\hline 2 & Rupture hépatique & 30 & $\begin{array}{r}a \\
b \\
c \\
\text { ad. } \\
\text { nad. }\end{array}$ & $\begin{array}{l}14 / 8 \\
80 \\
50 \\
1.99 \\
4.95 \\
6.94\end{array}$ & $\begin{array}{l}14 / 8 \\
80 \\
10 \\
- \\
-\end{array}$ & $\begin{array}{l}13 / 8 \\
80 \\
50 \\
- \\
-\end{array}$ & $\begin{array}{l}15 / 7 \\
80 \\
100 \\
0.80 \\
3.16 \\
3.96\end{array}$ \\
\hline 3 & Fracture du crane & 70 & $\begin{array}{r}\text { a } \\
\text { b } \\
\text { c } \\
\text { ad. } \\
\text { nad. } \\
\text { nad. + ad. }\end{array}$ & $\begin{array}{l}14 / 8 \\
70 \\
90 \\
2.44 \\
2.46 \\
4.80\end{array}$ & $\begin{array}{l}11 / 5 \\
80 \\
70 \\
4.41 \\
6.29 \\
10.70\end{array}$ & $\begin{array}{l}13 / 8 \\
70 \\
200 \\
3.19 \\
3.12 \\
6.31\end{array}$ & $\begin{array}{c}14 / 8 \\
70 \\
100 \\
= \\
=\end{array}$ \\
\hline 4 & Hémorragie externe & 56 & $\begin{array}{r}\text { a } \\
\text { b } \\
\text { c } \\
\text { ad. } \\
\text { nad. }\end{array}$ & $\begin{array}{l}8 / 0 \\
90 \\
30 \\
2.85 \\
4.18 \\
7.03\end{array}$ & $\begin{array}{c}11 / 6 \\
100 \\
65 \\
3.39 \\
4.08 \\
7.47\end{array}$ & $\begin{array}{c}12 / 6 \\
100 \\
10 \\
= \\
=\end{array}$ & $\begin{array}{c}14 / 8 \\
100 \\
100 \\
= \\
=\end{array}$ \\
\hline 5 & Hémorragie abdominale & 37 & $\begin{array}{r}a \\
b \\
c \\
\text { ad. } \\
\text { nad. } \\
\text { nad. + ad. }\end{array}$ & $\begin{array}{c}10 / 6 \\
120 \\
0 \\
7.40 \\
18.40 \\
25.80\end{array}$ & $\begin{array}{c}9 / 6 \\
140 \\
0 \\
11.87 \\
46.18 \\
58.05\end{array}$ & $\begin{array}{c}8 / 6 \\
160^{0} \\
= \\
=\end{array}$ & $\begin{array}{c}14 / 8 \\
120 \\
50 \\
- \\
-\end{array}$ \\
\hline 6 & $\begin{array}{l}\text { Fractures multiples } \\
\text { (bassin) }\end{array}$ & 18 & $\begin{array}{r}\mathrm{a} \\
\mathrm{b} \\
\mathrm{c} \\
\text { ad. } \\
\text { nad. } \\
\text { nad. + ad. }\end{array}$ & $\begin{array}{l}18 / 9 \\
160 \\
75 \\
1.28 \\
3.19 \\
4.47\end{array}$ & $\begin{array}{c}13 / 9 \\
130 \\
50 \\
0.86 \\
3.10 \\
3.96\end{array}$ & $\begin{array}{c}17 / 9 \\
120 \\
20 \\
= \\
-\end{array}$ & $\begin{array}{c}14 / 8 \\
100 \\
100 \\
= \\
-\end{array}$ \\
\hline 7 & $\begin{array}{l}\text { Fractures multiples } \\
\text { (bassin) }\end{array}$ & 61 & $\begin{array}{r}\mathrm{a} \\
\mathrm{b} \\
\mathrm{c} \\
\mathrm{ad} . \\
\text { nad. }\end{array}$ & $\begin{array}{l}12 / 7 \\
100 \\
20 \\
2.14 \\
4.53 \\
6.67\end{array}$ & $\begin{array}{l}13 / 7 \\
130 \\
15 \\
1.97 \\
3.48 \\
5.45\end{array}$ & $\begin{array}{c}8 / 0 \\
120 \\
30 \\
1.14 \\
2.81 \\
3.95\end{array}$ & $\begin{array}{c}14 / 7 \\
120 \\
50 \\
= \\
=\end{array}$ \\
\hline
\end{tabular}


TABLEAU II (suite)

\begin{tabular}{|c|c|c|c|c|c|c|c|}
\hline \multirow{2}{*}{$\begin{array}{l}\text { Patien } \\
\text { No }\end{array}$} & \multirow[b]{2}{*}{ Diagnostic } & \multirow[b]{2}{*}{ Age } & & \multicolumn{3}{|c|}{ Etat de choc } & \multirow{2}{*}{$\begin{array}{l}\text { Choc } \\
\text { terminé }\end{array}$} \\
\hline & & & & $1^{\mathrm{r} \theta} \mathrm{h}$. & $2^{\mathrm{me} \theta} \mathrm{H}$ & $3^{\mathrm{me}} \mathrm{H}$ & \\
\hline 8 & $\begin{array}{l}\text { Fractures multiples } \\
\text { (bassin) }\end{array}$ & 55 & $\begin{array}{r}\mathrm{a} \\
\mathrm{b} \\
\mathrm{c} \\
\text { ad. } \\
\text { nad. }\end{array}$ & $\begin{array}{l}13 / 8 \\
100 \\
75 \\
4.41 \\
3.25 \\
7.66\end{array}$ & $\begin{array}{l}14 / 8 \\
120 \\
175 \\
3.52 \\
3.68 \\
7.20\end{array}$ & $\begin{array}{l}15 / 8 \\
120 \\
100 \\
3.45 \\
3.33 \\
6.78\end{array}$ & $\begin{array}{l}16 / 8 \\
90 \\
50 \\
- \\
-\end{array}$ \\
\hline 9 & Hémorragie abdominale & 64 & $\begin{array}{r}\mathrm{a} \\
\mathrm{b} \\
\mathrm{c} \\
\text { ad. } \\
\text { nad. } \\
\text { nad. }\end{array}$ & $\begin{array}{c}4 / 0 \\
100 \\
0 \\
5.46 \\
9.65 \\
15.11\end{array}$ & $\begin{array}{c}11 / 5 \\
110 \\
0 \\
6.08 \\
8.99 \\
15.07\end{array}$ & $\begin{array}{c}4 / 0 \\
100 \\
0 \\
= \\
=\end{array}$ & $\begin{array}{c}14 / 8 \\
100 \\
4 \\
= \\
=\end{array}$ \\
\hline 10 & $\begin{array}{l}\text { Fractures multiples } \\
\text { (bassin) }\end{array}$ & 47 & $\begin{array}{r}\mathbf{a} \\
\mathbf{b} \\
\mathbf{c} \\
\text { ad. } \\
\text { nad. } \\
\text { nad. + ad. }\end{array}$ & $\begin{array}{c}10 / 5 \\
100 \\
0 \\
2.00 \\
2.62 \\
4.62\end{array}$ & $\begin{array}{c}10 / 5 \\
120 \\
50 \\
1.95 \\
2.47 \\
4.42\end{array}$ & $\begin{array}{l}12 / 7 \\
130 \\
100 \\
5.42 \\
5.50 \\
10.92\end{array}$ & $\begin{array}{c}14 / 8 \\
100 \\
50 \\
= \\
-\end{array}$ \\
\hline 11 & Hêmorragie gastrique & 65 & $\begin{array}{r}\text { a } \\
\mathbf{b} \\
\mathbf{c} \\
\text { ad. } \\
\text { nad. } \\
\text { nad. + ad. }\end{array}$ & $\begin{array}{c}11 / 6 \\
120 \\
0 \\
1.53 \\
2.85 \\
4.38\end{array}$ & $\begin{array}{c}13 / 6 \\
130 \\
0 \\
2.51 \\
5.69 \\
8.20\end{array}$ & $\begin{array}{c}9 / 5 \\
140 \\
100 \\
= \\
=\end{array}$ & $\begin{array}{l}16 / 9 \\
80 \\
80 \\
1.01 \\
1.34 \\
2.35\end{array}$ \\
\hline 12 & Choc septique & 48 & $\begin{array}{r}\mathbf{a} \\
\mathbf{b} \\
\mathbf{c} \\
\text { ad. } \\
\text { nad. } \\
\text { nad. + ad. }\end{array}$ & $\begin{array}{c}13 / 8 \\
120 \\
70 \\
3.85 \\
6.00 \\
9.85\end{array}$ & $\begin{array}{c}13 / 8 \\
120 \\
40 \\
2.28 \\
3.06 \\
5.34\end{array}$ & $\begin{array}{l}14 / 8 \\
120 \\
20 \\
1.93 \\
2.58 \\
4.51\end{array}$ & $\frac{\bar{z}}{\overline{4}} \overline{-}$ \\
\hline 13 & Rupture splénique & 69 & $\begin{array}{r}\mathrm{a} \\
\mathrm{b} \\
\mathrm{c} \\
\mathrm{ad} . \\
\text { nad. }\end{array}$ & $\begin{array}{l}16 / 10 \\
100 \\
100 \\
2.44 \\
4.05\end{array}$ & $\begin{array}{l}15 / 10 \\
100 \\
60 \\
1.81 \\
2.37\end{array}$ & $\begin{array}{c}14 / 9 \\
100 \\
100 \\
- \\
-\end{array}$ & $\begin{array}{l}15 / 10 \\
80 \\
70 \\
-\end{array}$ \\
\hline 14 & Hémorragie abdominale & 43 & $\begin{array}{r}\text { a } \\
\mathbf{b} \\
\mathbf{c} \\
\text { ad. } \\
\text { nad. } \\
\text { nad. + ad. }\end{array}$ & $\begin{array}{l}12 / 8 \\
80 \\
15 \\
1.08 \\
2.49 \\
3.57\end{array}$ & $\begin{array}{l}14 / 8 \\
80 \\
15 \\
1.07 \\
2.20 \\
3.27\end{array}$ & $\begin{array}{c}15 / 9 \\
100 \\
15 \\
= \\
=\end{array}$ & $\begin{array}{c}18 / 9 \\
100 \\
60 \\
= \\
-\end{array}$ \\
\hline & $\begin{array}{l}\text { Moyenne chez les } \\
\text { patients choqués }\end{array}$ & & $\begin{array}{r}\text { ad. } \\
\text { nad. } \\
\text { nad. + ad. }\end{array}$ & $\begin{array}{l}2.89 \\
5.19 \\
8.08\end{array}$ & $\begin{array}{r}3.30 \\
7.34 \\
10.64\end{array}$ & $\begin{array}{l}3.02 \\
3.46 \\
6.48\end{array}$ & $\begin{array}{l}0.91 \\
2.17 \\
3.08\end{array}$ \\
\hline & $\begin{array}{l}\text { Moyenne chez les } \\
\text { individus normaux }\end{array}$ & & $\begin{array}{r}\text { ad. } \\
\text { nad. } \\
\text { nad. }+ \text { ad. }\end{array}$ & & $\begin{array}{l}1.58 \\
2.35 \\
3.93\end{array}$ & & \\
\hline
\end{tabular}

$\mathrm{a}=$ pression artérielle en $\mathrm{cm} \mathrm{Hg}$.

$\mathrm{b}=$ fréquence cardiaque.

$c=$ diurèse horaire en cc.

Ad. = adrénaline en $\mu \mathrm{g} / \mathrm{L}$.

Nad. = Noradrénaline en $\mu \mathrm{g} / \mathrm{L}$. 
Ces patients manifestaient un état de choc marqué avec vasoconstriction très intense accompagnée, le plus souvent, de lividités; chez 10 d'entre eux, la diurèse horaire est devenue inférieure à $50 \mathrm{ml}$ dans le décours de l'affection, mais dans 5 cas seulement la pression artérielle systolique est tombée en dessous de $10 \mathrm{~cm} \mathrm{Hg.} \mathrm{Chaque} \mathrm{blessé} \mathrm{a,} \mathrm{bien} \mathrm{entendu,} \mathrm{reçu} \mathrm{le} \mathrm{traitement} \mathrm{qu'exigeait} \mathrm{son}$ état; il a été perfusé et transfusé selon les nécessités, sans cependant recevoir la moindre dose d'un médicament vasoconstricteur.

Les teneurs plasmatiques des catécholamines sont très variables selon les malades étudiés. Alors que le taux d'adrénaline le plus élevé, trouvé chez les témoins, est de $3.97 \mu \mathrm{g} / \mathrm{L}$ (moyenne: $1.58 \mu \mathrm{g} / \mathrm{L}$ ), 5 des 14 patients choqués dépassent ce chiffre. Pour ce qui est de la noradrénaline, le taux le plus élevé chez les témoins est de $4.80 \mu \mathrm{g} / \mathrm{L}$ (moyenne: $2.35 \mu \mathrm{g} / \mathrm{L}$ ), 6 des 14 patients choqués dépassent ce chiffre.

De plus, les chiffres moyens obtenus pour l'ensemble de lobservation sont nettement plus élevés chez les patients choqués que chez les témoins pour l'adrénaline $(3.30 \mu \mathrm{g} / \mathrm{L}$ contre $1.58 \mu \mathrm{g} / \mathrm{L})$ et la noradrénaline $(7.34 \mu \mathrm{g} / \mathrm{L}$ contre 2.35 $\mu \mathrm{g} / \mathrm{L})$ et pour la somme de A et NA $(10.64 \mu \mathrm{g} / \mathrm{L}$ contre $3.93 \mu \mathrm{g} / \mathrm{L})$.

Ainsi, une libération accrue des catécholamines se manifeste au cours du choc, témoin d'une stimulation intensive et prolongée du système orthosympathique. Cette stimulation semble porter non seulement sur la surrénale, mais de façon importante sur le système sympathique périphérique comme en témoigne le taux élevé de noradrénaline décelé dans le courant sanguin.

Cette hypercatécholaminémie régresse avec la disparition du choc jusqu'à aboutir aux teneurs relevées chez l'homme normal. Six heures après, on retrouve en effet chez 3 des 14 patients étudiés un taux d'adrénaline moyen de $0.91 \mu \mathrm{g} / \mathrm{L}$ (1.58 $\mu \mathrm{g} / \mathrm{L}$ chez les individus normaux), un taux de noradrénaline de $2.17 \mu \mathrm{g} / \mathrm{L}$ $(2.35 \mu \mathrm{g} / \mathrm{L}$ chez les sujets normaux) et un taux de catécholamines globales de $3.08 \mu \mathrm{g} / \mathrm{L}(3.93 \mu \mathrm{g} / \mathrm{L} \mathrm{chez}$ les individus normaux).

La relation entre l'hypercatécholaminémie et l'hypotension n'est pas absolument évidente. En effet, si l'on prend les cinq cas, $\mathrm{n}^{\circ} 3,5,9,11,12$ dont le taux global d'A + NA est de plus de $8 \mu \mathrm{g} / \mathrm{L}$ (soit plus du double de la moyenne observée chez les individus normaux), on constate que seuls les patients 5,9 et 11 ont présenté, pendant le décours de leur choc, une pression artérielle systolique inférieure à $9 \mathrm{~cm} \mathrm{Hg}$ alors que les patients 3 et 12 avaient des pressions artérielles systoliques supérieures à $11 \mathrm{~cm} \mathrm{Hg}$.

Par contre une réduction importante de la diurèse accompagne les états d'hypercatécholaminémie les plus élevés. En effet, les patients 5 et 9 qui ont atteint les taux d' ${ }_{\mathrm{A}}+\mathrm{NA}_{\mathrm{A}}$ les plus hauts, soit respectivement 58.05 et $15.11 \mu \mathrm{g} / \mathrm{L}$, ont souffert d'une anurie totale pendant le décours de leur choc.

\section{Catécholamines surrénaliennes}

L'examen comparatif des surrénales prélevées chez des individus normaux et au moment de la mort témoigne également de la stimulation intense que subit la surrénale au cours du choc. Le Tableau III rassemble les valeurs observées. Alors que dans les surrénales normales, les taux respectifs d'adrénaline, de noradrénaline et de catécholamines sont respectivement de $6.95,3.76,8.54 \mu \mathrm{g} / 10 \mathrm{mg}$, 
TABLEAU III

Teneur des catécholamines aU SEIN des surRénales PRÉlevées CHez les INDIVIdus À SYSTÈME CARDIOVASCULAIRE INTACT ET CHEZ LES POLYTRAUMATISÉS Catecholamine Contents in the Adrenal Glands of Men with Normal Cardiovascular System, and in Patients with Multiple Trauma

\begin{tabular}{|c|c|c|c|c|c|c|}
\hline & \multicolumn{3}{|c|}{ Surrénales normales } & \multicolumn{3}{|c|}{ Surrénales en cours de choc } \\
\hline & $\mathrm{A}(\mu \mathrm{g} / 10 \mathrm{mg})$ & NA $(\mu \mathrm{g} / 10 \mathrm{mg})$ & $\mathbf{A}+\mathbf{N A}$ & A $(\mu \mathrm{g} / 10 \mathrm{mg})$ & NA $(\mu \mathrm{g} / 10 \mathrm{mg})$ & $A+N A$ \\
\hline \multirow[t]{2}{*}{$\begin{array}{l}1 \\
2 \\
3 \\
4 \\
5 \\
\text { Moyenne }\end{array}$} & $\begin{array}{r}10.58 \\
7.11 \\
5.45 \\
6.45 \\
5.17\end{array}$ & $\begin{array}{l}5.10 \\
3.59 \\
3.56 \\
3.20 \\
3.37\end{array}$ & $\begin{array}{r}15.68 \\
10.70 \\
9.01 \\
9.65 \\
8.54\end{array}$ & $\begin{array}{l}3.89 \\
2.46 \\
2.78 \\
0.28\end{array}$ & $\begin{array}{l}2.97 \\
2.36 \\
3.27 \\
0.31\end{array}$ & $\begin{array}{l}6.86 \\
4.82 \\
6.05 \\
0.59\end{array}$ \\
\hline & 1e $\quad 6.95$ & 3.76 & 8.54 & 2.35 & 2.23 & 4.58 \\
\hline
\end{tabular}

après le choc ces taux deviennent $2.35,2.23,4.58 \mu \mathrm{g} / 10 \mathrm{mg}$. Le choc provoque une stimulation trop importante pour que les surrénales puissent compenser par une resynthèse immédiate les catécholamines qui quittent le parenchyme surrénalien.

Néanmoins, si le taux des catécholamines surrénaliennes est abaissé chez les patients morts en état de choc, il parait encore suffisant pour répondre aux besoins éventuels. Il en va de même pour la catécholaminémie qui, selon Hardaway $^{8}$ ne montre jamais d'insuffisance au cours du choc.

\section{Conclusions}

De l'ensemble de ces résultats, on peut conclure que le système orthosympathique est stimulé de façon intense chez l'individu en état de choc. Cette stimulation se marque par une hausse de la catécholaminémie sanguine accompagnée d'un appauvrissement relatif de la réserve en amines adrénergiques de la médullosurrénale. Néanmoins, l'efficacité clinique de cette stimulation n'est pas apparente dans chaque cas.

Du point de vue clinique, l'état de choc et l'hypercatécholaminémie ne sont pas nécessairement liés à une chute importante de la pression artérielle. Par contre, la réduction de la diurèse accompagne les états d'hypercatécholaminémie les plus marqués.

\section{RÉsuḾ́}

Le choc provoque une stimulation intense du système orthosympathique qui se traduit par une augmentation du taux des catécholamines sanguines et par un appauvrissement des catécholamines surrénaliennes. Chez 14 patients atteints de choc, le plus souvent traumatique, le taux de l'adrénaline et de la noradrénaline augmente dans des proportions appréciables pour atteindre des valeurs moyennes deux fois plus élevées que celles des individus normaux. Chez cinq patients morts en état de choc, le taux des catécholamines surrénaliennes est très inférieur au taux des surrénales normales. L’hypercatécholaminémie n'est pas nécessairement liée à l'hypotension artérielle. 